\title{
Prediction and Characterization of the Marcasite Phase of Iron Perni- tride under High Pressure
}

Zhongyu Wang,,$\uparrow$ Y Yucai Li, $\uparrow$ Hengtao Li,$\uparrow$ Ismail Harran, $\uparrow$ Mingzhen Jia,$\uparrow$ Hui Wang, $\uparrow$ Yuanzheng Chen $*, \dagger$ Hongyan Wang*,,$\uparrow$ and Nannan $\mathrm{Wu}^{\xi}$

${ }^{\dagger}$ School of Physical Science and Technology, Key Laboratory of Advanced Technologies of Materials, Ministry of Education of China, Southwest Jiaotong University, Chengdu 610031, Peoples Republic of China

\$ Tourism and Urban-Rural Planning College, Chengdu University of Technology, Chengdu 610031, Peoples Republic of China

${ }^{\xi}$ Inner Mongolia University of Science \&Technology, School of Science, Baotou 014000, Peoples Republic of China

\begin{abstract}
:
Using the unbiased structure searching CALYPSO techniques and the first-principles calculations, we predicted a phase transition in iron pernitride $\left(\mathrm{FeN}_{2}\right)$ at $22 \mathrm{GPa}$ from the hexagonal $R-3 m$ structure to an orthorhombic Pnnm structure. Although the Pnnm structure is a well-known marcasite phase of transition-metal pernitrides, to our knowledge, this is the first report about the pressure-induced phase transition to the Pnnm structure in the $\mathrm{FeN}_{2}$ compound. The dynamically and mechanically stable for the Pnnm phase are evaluated, further confirming this phase transition. By assessing the elastic property of the Pnnm structure, the result indicates that it displays excellent brittle and hardness characters. Viewing it's structure, a dinitrogen unit and a coordinating iron atom characterized by N-sharing six-fold FeN 6 octahedrons are found. Based on the quantum-chemical analysis, the dinitrogen unit was formulated as a quasi-molecular double-bonded $\mathrm{N}_{2}{ }^{2-}$ and a mixed covalent $\mathrm{N}-\mathrm{N}$ and $\mathrm{Fe}-\mathrm{N}$ bonding nature was identified in the structure. These structural and covalent bonding characters play a crucial role to its excellent mechanical properties. The present results extend the well-known marcasite phase in other transition-metal pernitrides to the $\mathrm{FeN}_{2}$ compound and provide a perspective toward the understanding of the synthesis and chemical bonding of $\mathrm{N}$-rich $\mathrm{FeN}_{2}$ under high pressure.
\end{abstract}

Keywords: Iron-nitrides compounds, Dinitrogen, High pressure, Particle Swarm Optimizatio 


\section{Introduction}

The binary iron-nitrides (Fe-N) system has been investigated most intensively since 19 century [1]. A series of low nitrogen content compounds $\left(\mathrm{Fe}_{2} \mathrm{~N}, \mathrm{Fe}_{3} \mathrm{~N}, \mathrm{Fe}_{4} \mathrm{~N}, \mathrm{Fe}_{8} \mathrm{~N}\right)$ successively synthesized starting from their constituent elements [2-4]. A N-rich FeN compound in which a nitrogen ratio is up to $50 \%$ was reported by experimental studies [5]. These synthesis spurred the endeavors in search for N-rich Fe$\mathrm{N}$ compounds. However, up to now, there no other Fe-N compound that its nitrogen content exceeds the FeN compound can be synthesized by experiment. Recently, an N-rich iron pernitride $\left(\mathrm{FeN}_{2}\right)$ crystallizing in the space group $R-3 m$ at $17 \mathrm{GPa}(1000 \mathrm{~K})$ is predicted theoretically [6]. Its structural, electronic and dynamical stability have been further elaborated by Gupta using a spin polarized first-principles study [7]. This new crystalline phase of $\mathrm{FeN}_{2}$ compound has been obtained by assuming the parent metal under the pressure conditions, which opens and arouses a research of exploring pressure-induced structural phases of $\mathrm{FeN}_{2}$.

The $\mathrm{FeN}_{2}$ compound, as a member of transition-metal pernitrides family, is confirmed to have similar high-pressure phases of transition-metal pernitrides $\mathrm{AN}_{2}(\mathrm{~A}=\mathrm{Os}, \mathrm{Ru}, \mathrm{Ir}$, and $\mathrm{Pt}$ ) [8-9]. Due to Fe belonging to the same group as $\mathrm{Os}$ and $\mathrm{Ru}$ in the periodic table of elements, $\mathrm{FeN}_{2}$ was recognized to also have a marcasite (orthorhombic, Pnnm) structure existing in the case of $\mathrm{OsN}_{2}$ and $\mathrm{RuN}_{2}$ at high pressure [10-12]. But, this viewpoint has not yet confirmed in theory or experiments.

In order to explore the stable high-pressure structure of $\mathrm{FeN}_{2}$, the phase stabilities of various stoichiometric $\mathrm{Fe}_{\mathrm{x}} \mathrm{N}_{1-\mathrm{x}}(0<\mathrm{x}<1)$ compounds at 0 and $50 \mathrm{GPa}$ are investigated systematically to obtain the formation enthalpy of $\mathrm{FeN}_{2}$. A combination of the unbiased structural search based on a particle-swarm optimization (PSO) algorithm [13] and the first-principles density functional total energy calculation is used to explore the entire configuration space. The effectiveness of the method has been demonstrated by recent successes in predicting the high-pressure structures of various systems [14-18]. The highpressure insulating Aba2-40 (Pearson symbol $o C 40$ ) structure of $\mathrm{Li}$, the two low-pressure monoclinic structures of $\mathrm{Bi}_{2} \mathrm{Te}_{3}$, and the orthorhombic Imm2 structure of $\mathrm{FeTi}_{3} \mathrm{O}_{7}$ predicted by this method have been confirmed by experiments [16-18]. Here we report a Pnnm structure of FeN $\mathrm{N}_{2}$ as a well-known marcasite phase in the transition-metal pernitrides. Theoretical calculations reveal that the Pnnm structure is more stable than the reported $R-3 m$ structure at pressures above $22 \mathrm{GPa}$. A dinitrogen unit and a coordinating iron atom characterized by $\mathrm{N}$-sharing six-fold $\mathrm{FeN}_{6}$ octahedrons are found in the structure. The quasi-molecular double-bonded $\mathrm{N}_{2}{ }^{2-}$ pernitride units and the mixed covalent $\mathrm{N}-\mathrm{N}$ and Fe-N bonding nature were identified. By the calculations of bulk moduli, shear moduli, Young's moduli, Poisson's ratio and Vickers hardness, it indicates that the Pnnm structure possesses excellent mechanical properties. 


\section{Methods}

The structure searching simulations were performed through the swarm-intelligence based CALYPSO method enabling a global minimization of free energy (at $0 \mathrm{~K}$, it reduces to enthalpy) surfaces merging ab initio total energy calculations as implemented in the CALYPSO code [13]. The method is specially designed for global structural minimization unbiased by any known structural information, and has been benchmarked on various known systems [19-21]. To find the $\mathrm{FeN}_{2}$ structure lying the lowest in energy, the structures of $\mathrm{FeN}_{2}$ and its decomposition various $\mathrm{Fe}_{\mathrm{x}} \mathrm{N}_{1-\mathrm{x}}(0<\mathrm{x}<1)$ compounds with simulation cell sizes of 1-4 formula units (f.u.) at 0 and $50 \mathrm{GPa}$ were searched. The underlying ab initio electronic structure calculations and the geometry relaxations were performed in the framework of density functional theory (DFT) within the generalized gradient approximation and the frozen-core all-electron projectoraugmented wave (PAW) method [22-23] implemented in the Vienna ab Initio Simulation Package (VASP) code [24]. The PAW pseudopotentials with $3 d^{7} 4 s^{1}$ and $2 s^{2} 2 p^{3}$ valence electrons were adopted for $\mathrm{Fe}$ and $\mathrm{N}$, respectively. The kinetic energy cutoff for the plane-wave basis set is taken as $700 \mathrm{eV}$. The $11 \times 9 \times 17,16 \times 16 \times 4$ Monkhorst-Pack [25] grid for the k-points sampling are used for the Pnnm and $R-3 m$ structures, respectively. The convergence of these parameters is well checked, with the uncertainty in the total energy less than $1 \mathrm{meV}$ per atom. The phonon calculations and the quasi-harmonic freeenergy calculations were carried out by using a finite displacement approach through the PHONOPY code [26]. The macroscopic stress in the solid can be computed for a small strain by the use of the strain-stress method. The elastic moduli can be derived from the strain-stress relationship [27]. After obtaining the single-crystal elastic mouduli, polycrystalline properties such as bulk moduli, shear moduli, and ductile/brittle are calculated by using the Voigt-Reuss-Hill approximation [27-28]. Vicker's hardness is formulated by Tian et al [29-31]. A quantum-chemical analysis is carried out by means of the crystal orbital Hamilton population (COHP) method ${ }^{31}$ using the LOBSTER program [32-33].

\section{Results and discussions}

Structural predictions for the $\mathrm{FeN}_{2}$ compound was performed using the CALYPSO code with simulation cells contaning 1-4 f.u. at 0-50 GPa. We typically generated several hundred structures to ensure the convergence of the global structural searches. In these structures, the energetic stable structures mainly contain dinuclear $\mathrm{N}_{2}$ units. In this structural model, we not only successfully found the typical $R-3 m$ structure of $\mathrm{FeN}_{2}$ predicted by Richard Dronskowski [6] but also predicted an orthorhombic Pnnm structure with lowest enthalpies at $50 \mathrm{GPa}$. By comparing the enthalpy curves in Figure 1a, it is found that the Pnnm structure is even more stable than the $R-3 m$ structure at pressures above $11 \mathrm{GPa}(0 \mathrm{~K})$. 
As well known, magnetism plays a central role in iron and its compounds. Therefore it is necessary to check the role of magnetism on the affect of the energy balance between the $R-3 m$ and Pnnm structures. We therefore performed the enthalpy calculations after considering spin polarized mode in Figure 1b. As shown in the Figure $1 b$, the enthalpy difference curves show that the lowest-energy $\mathrm{FeN}_{2}$ phase adopts $R-3 m$ at low pressure (13-22 GPa) and transform into the Pnnm structure at above $22 \mathrm{GPa}$. It found that the spin polarization effect shifted the phase transition pressure from $11 \mathrm{GPa}$ to $22 \mathrm{GPa}$.
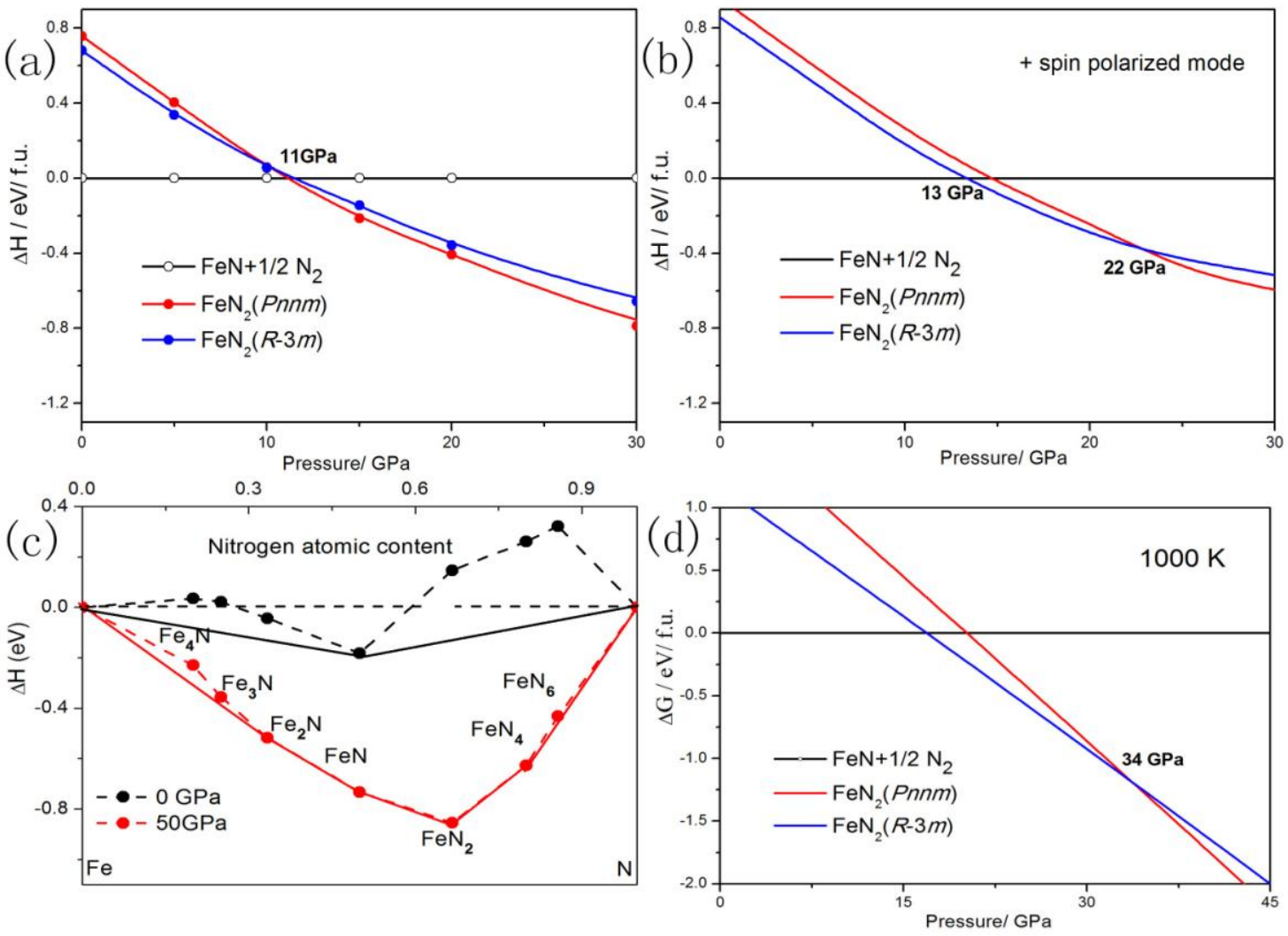

Figure 1. (a) Enthalpy curves (relative to the decomposition enthalpies of $\mathrm{FeN}+1 / 2 \mathrm{~N}_{2}$ ) of various structures as function of pressure in $\mathrm{FeN}_{2}$ compound. Enthalpies are given per formula unit. (b) The enthalpy curves ( relative to the decomposition enthalpies of $\mathrm{FeN}+1 / 2 \mathrm{~N}_{2}$ ) after considering spin polarized mode. (c) Formation enthalpies $(\Delta \mathrm{H})$ of various Fe-N compounds with respect to decomposition into constituent elemental solids at 0 and $50 \mathrm{GPa}$ in the convex hull. The convex hulls are shown by solid lines. Dotted lines through the neighboring points residing above the convex hull are guides for the eye. (d) The Gibbs free energy-pressure diagram of $\mathrm{FeN}_{2}$

$$
(\mathrm{T}=1000 \mathrm{~K})
$$

The estimation of the heat of formation is not trivial, thus we need to compare the enthalpy of the $\mathrm{FeN}_{2}$ structure with its combination of possible starting materials $\mathrm{Fe}_{4} \mathrm{~N}, \mathrm{Fe}_{3} \mathrm{~N}, \mathrm{Fe}_{2} \mathrm{~N}, \mathrm{FeN}_{4}, \mathrm{FeN}_{6}$ with $\mathrm{N}_{2}$. The formation enthalpies of these compounds were calculated with respect to the decomposition into Fe and $\mathrm{N}$, where the enthalpies are obtained for the most stable structures as searched by the CALYPSO 
method at the desired pressures. The results were depicted to a convex hull shown in Figure 1c. The convex hull of the enthalpies of formation of Fe-N compound is constructed to account for all possible decompose routes at 0 and $50 \mathrm{GPa}$. Viewing the convex hull, it obviously shows that the most thermodynamically stable compound transforms from $\mathrm{FeN}$ at $0 \mathrm{GPa}$ to $\mathrm{FeN}_{2}$ at $50 \mathrm{GPa}$. The result confirms that the $\mathrm{FeN}_{2}$ compound can be synthesized at $50 \mathrm{GPa}$.

To take account for the temperature effects, we performed quasi-harmonic Gibbs free energy calculations using the finite-displacement method. The Gibbs free energy-pressure diagram for the synthesis of $\mathrm{FeN}_{2}$ at temperature of $\mathrm{T}=1000 \mathrm{~K}$ was performed and shown in Figure 1d. We found that the Pnnm phase synthesis of $\mathrm{FeN}_{2}$ at $1000 \mathrm{~K}$ needs a higher pressure (> $\left.34 \mathrm{GPa}\right)$ than the value ( 22 GPa ) at $0 \mathrm{~K}$. A similar trend of pressure dependence for the $R-3 m$ phase can be extracted from our data. These phenomenon manifest that temperatures have negative effect on $\mathrm{FeN}_{2}$ synthesis.

It is known that the Gibbs free energy reduces to the enthalpy $\mathrm{H}=\mathrm{U}+\mathrm{PV}$ at $0 \mathrm{~K}$, where $\mathrm{U}, \mathrm{P}$ and $\mathrm{V}$ are the static energy, pressure and volume per formula unit, respectively. The high-pressure competition between $U$ and PV dominates the phase transitions. A sphere packing efficiency calculations show that the Pnnm structure (11.5\%) is more densely packed than the $R-3 m$ structure $(10.7 \%)$ at $50 \mathrm{GPa}$, with a less volume. The less volume is critical to the lower enthalpy for the stabilization of Pnnm phase. Moreover, dynamical structural stability of the Pnnm structure was investigated by calculating phonon dispersion curves. No imaginary frequency was found for the structure, which indicated that it is dynamically stable.

The Pnnm structural lattice constants generated in the optimization at $25 \mathrm{GPa}$ are $\mathrm{a}=3.8015 \AA$, $\mathrm{b}=$ $4.5258 \AA$, and $\mathrm{c}=2.4840 \AA$, with Fe atoms on the Wyckoff sites $2 c(0,1 / 2,1)$, being different from in a common $2 a$ sublattice of metal atom in the marcasite type of $\mathrm{AN}_{2}$ [34-35], and $\mathrm{N}$ atoms on the site $4 g$ $(x, y)$ with two internal parameters $x$ and $y(x=0.13016, y=0.09678)$. Thus it can be regarded as an isostructure of the marcasite type. Calculations at different pressure confirm that there is no important effect of the pressure on the atomic positions of the atoms in the Pnnm structure. In the structure, it contains a dinitrogen unit with an N-N distance of $d_{1}=1.322 \AA$, and the coordination of the Fe atoms is surrounded by the dinitrogen units (Figure 2a). Each Fe atom has two nearest and four next-nearest neighboring $\mathrm{N}$ atoms with Fe-N distances of $d_{2}=1.891 \AA$ and $d_{3}=1.926 \AA$, respectively. When the pressure is increasing to $50 \mathrm{GPa}$, the dinitrogen $\mathrm{N}-\mathrm{N}$ distance is short to $1.317 \AA$ and the $\mathrm{Fe}-\mathrm{N}$ bond distances are compressed to $1.857 \AA$ and $1.891 \AA$, respectively. In the studied pressure range of Figure $2 \mathrm{~b}$, it is found that the dinitrogen $\mathrm{N}-\mathrm{N}$ distance has a small change while the Fe-N distances decrease monotonically as pressure increases. This result manifests the pressure have an important effect on the Fe-N bond but less 
effect the N-N bond. Polyhedral views (Figure 2a) of the Pnnm structure is similar to the $R-3 m$ structure with the same coordination number forming six-fold bond $\mathrm{FeN}_{6}$ octahedron, but it contains longer dinitrogen units and closer-packed iron, in contrast with the cubic layers hexagonal $R-3 m$ structure [6].

(a)

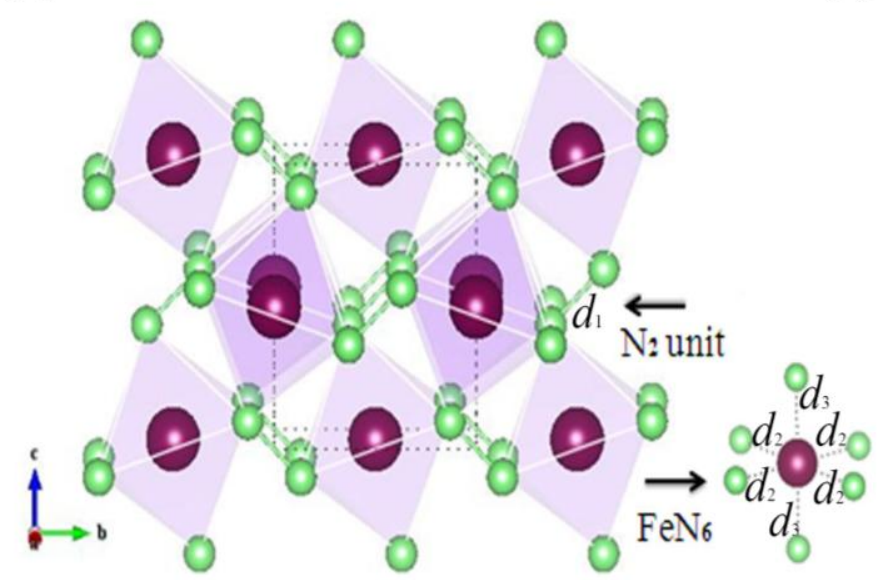

(b)

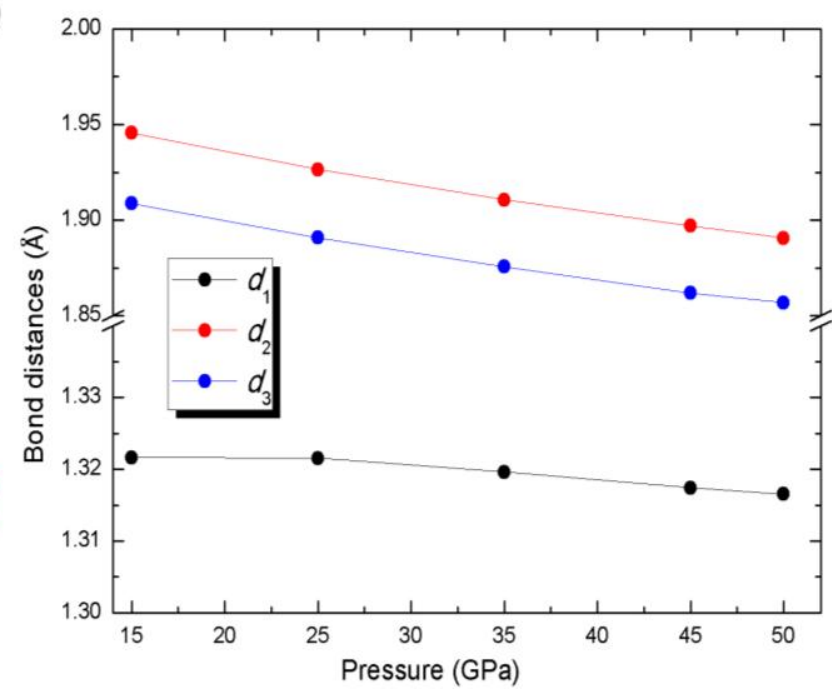

Figure 2. Crystal structures of the orthorhombic Pnnm structure in $\mathrm{FeN}_{2}$, a structural unit of 6-fold bonded $\mathrm{FeN} 6$ octahedrons. The blue and purple spheres indicate Fe and $\mathrm{N}$ atoms, respectively.

In the following, we are going to study the mechanical stability of the Pnnm structure under high pressure. Based on the Born criteria [36-37], the case of an orthorhombic crystal with six $C_{i j}$ elastic moduli, the mechanical stability at zero pressure requires that

$$
\begin{aligned}
& C_{11}>0, C_{44}>0, C_{55}>0, C_{66}>0 \\
& C_{11} C_{22}>C_{12}{ }^{2} \\
& C_{11} C_{22} C_{33}+2 C_{12} C_{13} C_{23}-C_{11} C_{23}{ }^{2}-C_{22} C_{13}{ }^{2}-C_{33} C_{12}{ }^{2}>0
\end{aligned}
$$

To study the mechanical stability of the orthorhombic crystal under pressure, such Eqs. (1) have to be modified to include the particular case when the external load is different from zero. Referring to the reports [36-37] about a detailed explanation of how the Born stability criteria must be modified when the solid is subject to a external load, we obtain the general stability criteria valid when the orthorhombic crystal is subjected to an external hydrostatic pressure $P$ in the following form (2):

$C_{11}-P>0, C_{44}-P>0, C_{55}-P>0, C_{66}-P>0$

$\left(C_{11}-P\right)\left(C_{22}-\mathrm{P}\right)>\left(\mathrm{C}_{12}+P\right)^{2}$

$\left(C_{11}-P\right)\left(C_{22}-P\right)\left(C_{33}-P\right)+2\left(C_{12}+P\right)\left(C_{13}+P\right)\left(C_{23}+P\right)-\left(C_{11}-P\right)\left(C_{23}+P\right)^{2}-\left(C_{22}-P\right)\left(C_{13}+P\right)^{2}-\left(C_{33}-P\right)\left(C_{12}+P\right)^{2}>0$

Figure 3 a shows the evolution of the calculated $C_{i j}$ of the Pnnm structure with pressure. It can be seen that all $C_{i j}$ elastic moduli increase monotonically as pressure increases. In the studied pressure range, we 
check the Pnnm structure whether satisfies Eqs. (2) for all pressures or not. It is found that all the above criteria are satisfied for the Pnnm structure. This fact indicates that the Pnnm phase is mechanically stable at the considered pressure, as expected.

We calculated the each axis of the Pnnm phase as a function of pressure to analyze the compressibility of each axis with pressure. As shown in Figure 3b, it is found that the $b$ and $c$ axis decrease as pressure increases while the $a$ axis almost keeps a constant. The $a$ axial compressibility $(0.23 \%)$ is less than that of the $b(0.35 \%)$ and $c$ axis $(0.34 \%)$ in the studied pressure range. This fact indicates that the $a$ axis exhibits stronger resistance to linear compression than the $b$ and $c$ axis. In order to research the mechanical properties of the Pnnm structure, the elastic moduli (B, G, E) and Poisson's ratio (v) have been calculated at $25 \mathrm{GPa}$ in the Table 1. The bulk moduli (B) from the elastic constants is calculated to have a value of $385 \mathrm{GPa}$. In addition, a fit to the calculated E-V points (Figure 3c) using a Birch Murnaghan EOS [38] gives a bulk moduli ( $\left.\mathrm{B}^{\prime}\right)$ values of $357 \mathrm{GPa}$. This $\mathrm{B}^{\prime}$ value compares well with the $\mathrm{B}$ value with small differences within the fitting errors. It is also important to notice that $\mathrm{B} / \mathrm{G}=1.48$. The critical $\mathrm{B} / \mathrm{G}$ value for ductile and brittle materials is 1.75 , indicating that the studied Pnnm structure of FeN 2 compound is brittle material. Regarding the effects of pressure on the brittleness of the Pnnm structure, we found that $\mathrm{B} / \mathrm{G}$ increases under pressure reaching at $50 \mathrm{GPa}$ values that is increasing to 1.55 . This fact indicates that under compression the brittleness of Pnnm structure become weak. Additionally, we calculated the Poisson's ratio $v$ defined as $v=E / 2 G-1$ which is an important physical quantity related to the stability of the crystal against shear. The calculated value $v$ is 0.22 a value smaller than the Poisson's ratio $\sim 0.3$ in cubic BN. It means that the stable of the Pnnm structure against shear can't be compatible to that of cubic BN.

Table 1. Calculated the bulk moduli B (GPa), shear moduli G (GPa), young's moduli E (GPa), Vickers hardness Hv (GPa) and Poisson's ratio (v) from the elastic constants and the bulk moduli B' (GPa) using a Birch-Murnaghan EOS for the Pnnm phase at $25 \mathrm{GPa}$

\begin{tabular}{c|ccccccc}
\hline Structure & $B$ & $B^{\prime}$ & $G$ & $B / G$ & $E$ & $H v$ & $v$ \\
\hline Pnnm & 385 & 357 & 259 & 1.48 & 634 & 26.3 & 0.22 \\
\hline
\end{tabular}

As known, most common hard transition-metal pernitrides crystallize have bulk moduli comparable with those of the traditional superhard materials. For example, the measured bulk moduli (B $=428 \mathrm{GPa})$ of $\mathrm{IrN}_{2}$ is second only to that of diamond (K=440 GPa) [39]. The bulk moduli or shear moduli can be a measure of the hardness in an indirect way. Note that the high bulk moduli for the Pnnm phase of FeN 2 indicates it is likely to be hard materials. The value is close to the bulk moduli ( $\mathrm{B}=368 \mathrm{GPa})$ of 
$\mathrm{Fe}_{3} \mathrm{Mo}_{3} \mathrm{~N}$, thus pointing to a very similar resistance to plastic deformations [38]. Employing the Simunek's Method, a theoretical Vickers hardness of $26.3 \mathrm{GPa}$ for the Pnnm phase is estimated, which is close to that of ternary nitrides $\left(\mathrm{Ni}_{2} \mathrm{Mo}_{3} \mathrm{~N}, \mathrm{Fe}_{3} \mathrm{Mo}{ }_{3} \mathrm{~N}\right)$ and other transition-metal pernitrides $\mathrm{AN}_{2}$ such as $\mathrm{OsN}_{2}[34-35,38]$. Such excellent mechanical properties would like to relate the incompressible with the dinitrogen units as found in other transition-metal pernitrides $\mathrm{AN}_{2}$. As charge transfer from A to $\mathrm{N}$ controls the length of the $\mathrm{N}-\mathrm{N}$ bond, resulting in a quite uncompressible $\mathrm{N}-\mathrm{N}$ bond, dominantly by providing Coulomb repulsion between the pairing $\mathrm{N}$ atoms.

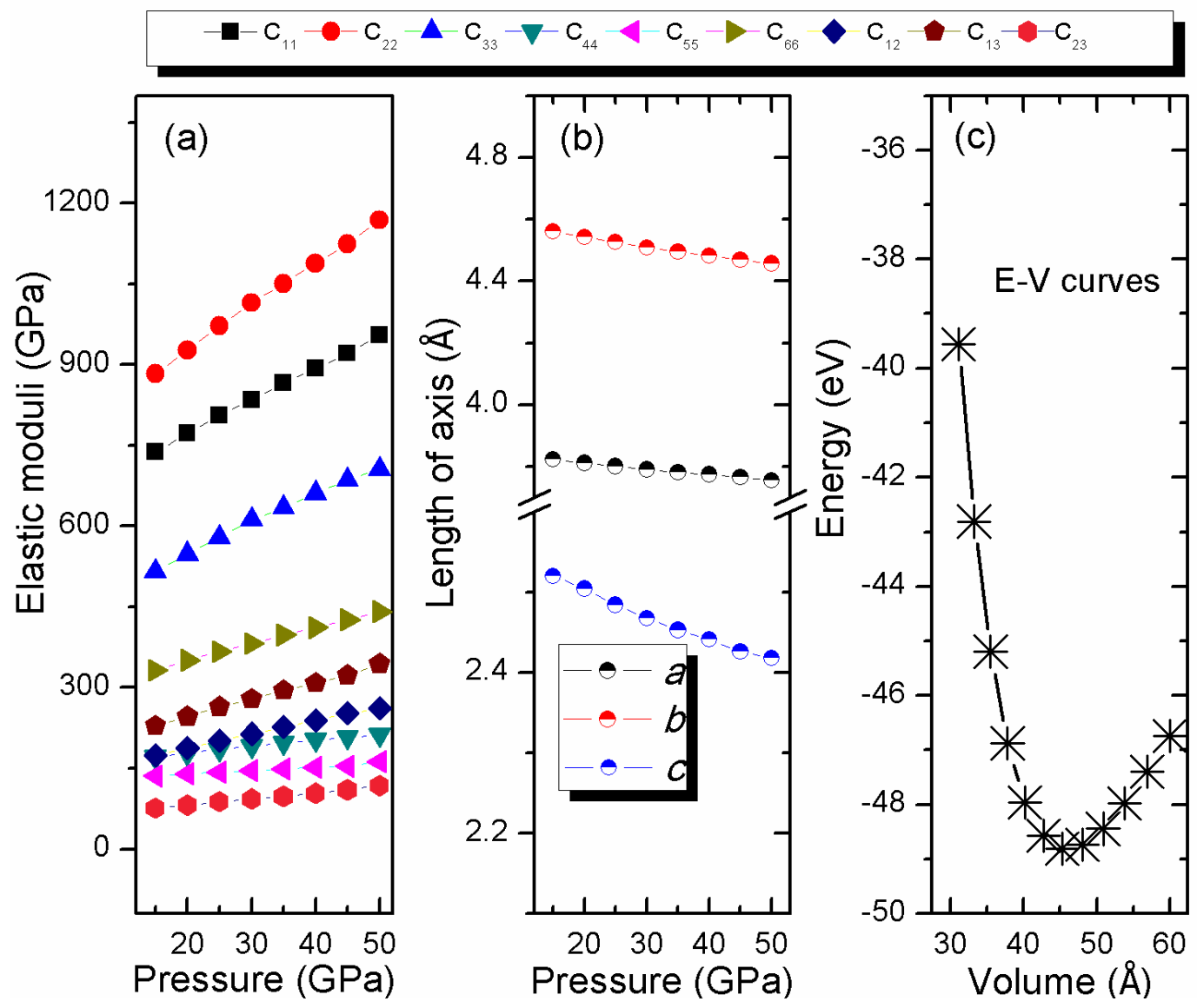

Figure 3. (a) Pressure dependence of the theoretical elastic moduli of the Pnnm structure at 25 GPa. (b) Calculated the each axis of the Pnnm phase as a function of pressure. (c) Total-energy versus volume from ab initio calculations for the Pnnm structure.

Return to the dinitrogen units, we note that it's N-N distance $(\sim 1.32 \AA)$ in the Pnnm structure is longer than the double bond $(1.27 \AA$ ) in the $R-3 m$ structure [6] and the $\mathrm{N} \equiv \mathrm{N}$ triple bond $(1.10 \AA)$ in the neutral $\mathrm{N}_{2}$, and smaller than the $\mathrm{N}-\mathrm{N}$ single bond $\left(1.47 \AA\right.$ ) that is presumably present in $\mathrm{N}_{2}^{4-}$ of $\mathrm{PtN}_{2}$ and $\mathrm{OsN}_{2}$ [9-10]. The N-N distance is nearly identical to the single $\mathrm{C}-\mathrm{C}$ bond $(1.31 \AA)$ of the $\mathrm{C}_{2}^{4-}$ unit in $\mathrm{ThC}_{2}$ [40], which directly shows that the Pnnm structure of $\mathrm{FeN}_{2}$ compound maybe have the $\mathrm{N}_{2}{ }^{4-}$ unit. 
However, a similar N-N distance (1.34-1.35 $\AA$ ) of $\mathrm{N}_{2}{ }^{2-}$ units was found in the dubbed $\mathrm{Li}_{2} \mathrm{Ca}_{3} \mathrm{~N}_{6}$ [41]. Even if the N-N distance increases to $1.46 \AA$ for $\mathrm{TiN}_{2}$, the $\mathrm{N}_{2}{ }^{2-}$ units still present in the compound. Therefore it is not safely to regard the dinitrogen units of the Pnnm structure as an $\mathrm{N}_{2}{ }^{4-}$ or $\mathrm{N}_{2}{ }^{2-}$ unit only judging from the N-N distances. The charge distribution is more appropriate to judge the dinitrogen units as $\mathrm{Fe}^{2+} \mathrm{N}_{2}{ }^{2-}$ or $\mathrm{Fe}^{4+} \mathrm{N}_{2}{ }^{4-}$. The Bader charge analysis [42] reveals that about $1.6 e$ from Fe atom transfers to the dinitrogen units in the Pnnm structure, which is close to the value (approximately 1.5 e) of the $R-3 m$ structure. The non-integer charges are due to the fact that the charges are calculated by partitioning space according to its zero-flux surfaces. The same situation is found in a typical ionic crystal $\mathrm{Na}^{1+} \mathrm{Cl}^{1-}$, in which the $\mathrm{Na}$ transfer charge is around $0.8 e$ using the Bader charge analysis. Therefore, it is plausible to recognize that the $\mathrm{N}_{2}{ }^{2-}$ units is present in the Pnnm structure.

Another indirect way to subject to proof the $\mathrm{N}_{2}{ }^{2-}$ units is given by a quantum-chemical analysis of the extended Pnnm structure of $\mathrm{FeN}_{2}$ indeed yields the antibonding states within the quasi-molecular nitrogen dimer. Figure 4 offers the COHPs for the Fe-Fe, Fe-N and the $\mathrm{N}-\mathrm{N}$ bonding inside $\mathrm{FeN}_{2}$. In the N-N combination, the antibonding states are found between -4 and $+0.5 \mathrm{eV}$, which differs from that in $\mathrm{PtN}_{2}$ completely filled [43]. Just like for $\mathrm{BaN}_{2}$, the nitrogen $-\pi_{\mathrm{p}} *$ levels in $\mathrm{FeN}_{2}$ are half-filled so that the pernitride unit can be formulated as $\mathrm{N}_{2}{ }^{2-}$, as above already suggested by the charge analysis. By comparing with the $R-3 m$ structure, the $R-3 m$ structure with the $\mathrm{N}_{2}{ }^{2-}$ units has similar COHP feature [6], which supported that the dinitrogen units of the Pnnm structure is regard as the $\mathrm{N}_{2}{ }^{2-}$ unit. Moreover, the strong bonding states also exist in the Pnnm structure. Besides a strongly bonding N-N bond behavior below the $-4.5 \mathrm{eV}$, there is a Fe-N combination bonding state in the entire occupied region. These bonding states of the occupied crystal orbitals reveal a mix Fe-N and N-N chemical bonding in the Pnnm structure. 

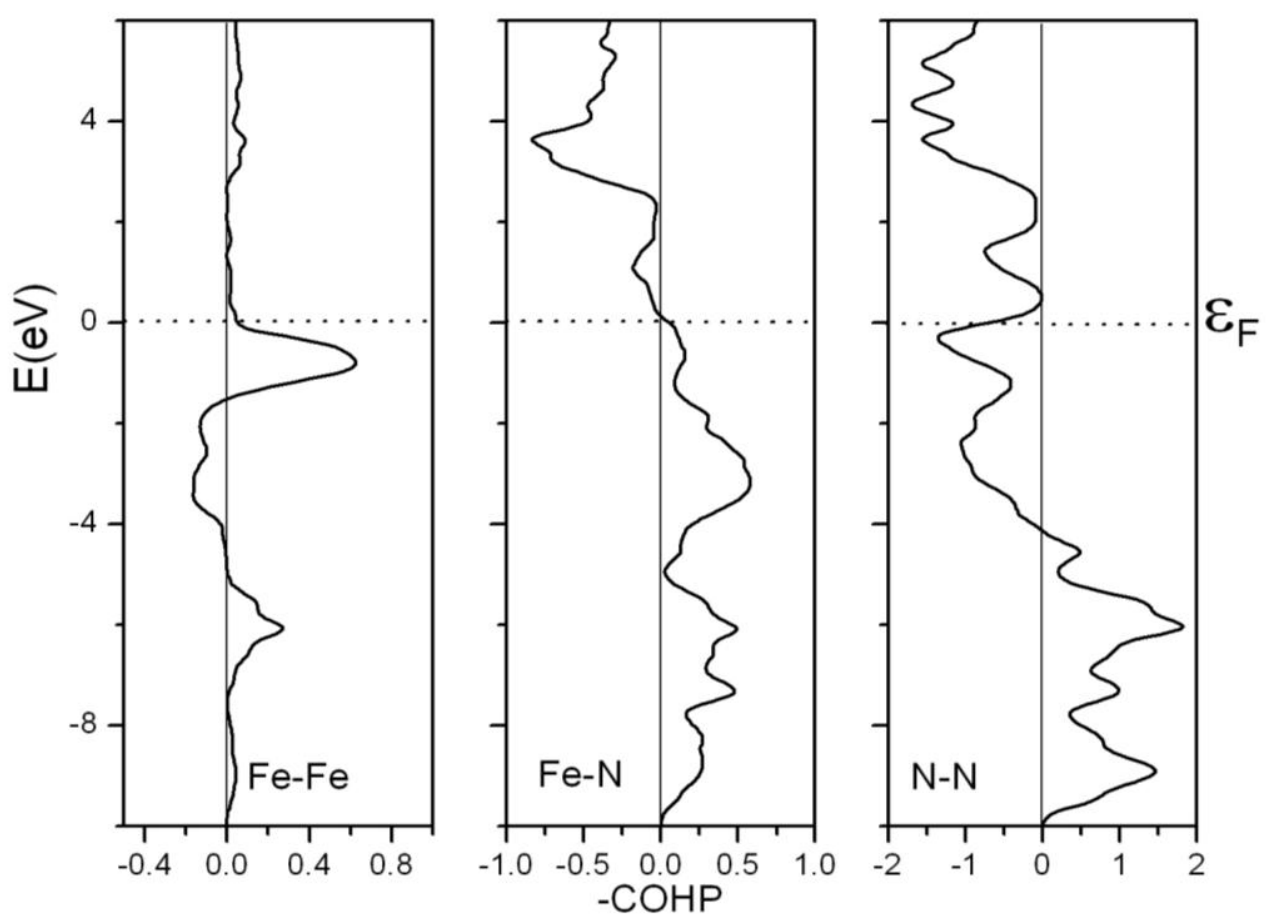

Figure 4. Crystal orbital Hamilton population (COHP) analysis of $\mathrm{Fe}-\mathrm{Fe}, \mathrm{Fe}-\mathrm{N}$, and $\mathrm{N}-\mathrm{N}$ bonding interactions within the Pnnm structure

The electronic band structure and partial density of states (PDOS) of the Pnnm structure were calculated (Figure 5). The result shows that $\mathrm{FeN}_{2}$ exhibit metallic features. This is in agreement with the $\mathrm{OsN}_{2}$ and $\mathrm{RuN}_{2}$, but differs from $\mathrm{IrN}_{2}$ and $\mathrm{PtN}_{2}$ which are nonmetallic. From PDOS of Figure 5, we noticed that $\mathrm{Fe} d$ orbital has hybridization with $\mathrm{N} p$ orbital, indicating a covalent bonding between Fe and $\mathrm{N}$. Fe belongs, like $\mathrm{Os}, \mathrm{Ru}$, to the transition metal series and just has electrons less than Os and $\mathrm{Ru}$, so a same metallic character would be compatible with electron counting arguments. To probe the role of Fe played in the electronic structures, we calculated the band structure of a hypothetical $\mathrm{Fe}_{0} \mathrm{~N}_{2}$ system by removing Fe out of the lattice, where $\mathrm{N}$ sublattice remains unchanged. A uniform compensated background charge $(8 \mathrm{e} / \mathrm{Fe})$ is applied to preserve the total valence electrons of the system. The resultant band structure (red dash lines in Figure 5) isn't essentially similar to the realistic one of FeN 2 . The indication is that the Fe atoms not only act as electron donors but also bond with $\mathrm{N}$, consistent with the analyses of COHP and PDOS as described above. One might conclude that the correlations the mechanical properties with the Fe action: acting as electron donors to the antibonding orbital help stretch the N-N covalent bond; acting bond with $\mathrm{N}$ increase the covalence of system, which involving covalency are the source of the mechanical properties. This reasoning can be also found to hold true for other transition-metal carbides and nitrides [31, 44-45]. 


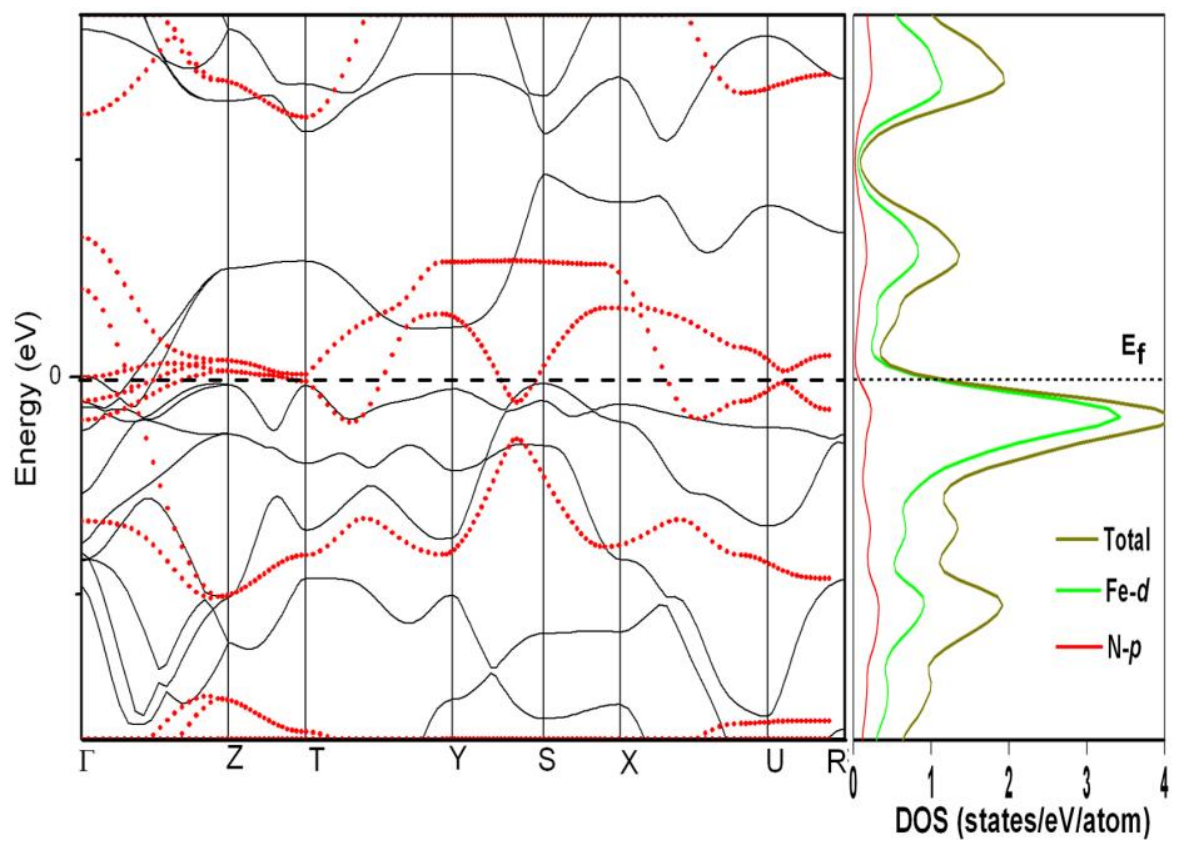

Figure 5. Electronic band structure and PDOS for the Pnnm phase at $25 \mathrm{GPa}$. In the left panel, the black solid lines are electronic band structure of $\mathrm{FeN}_{2}$; the red dashed lines represent the band structure of the $\mathrm{N}$ sublattice $\left(\mathrm{Fe}_{0} \mathrm{~N}_{2}\right)$ with a uniform compensated background charge; the $\Gamma\left(\begin{array}{lll}0 & 0 & 0\end{array}\right), \mathrm{Z}\left(\begin{array}{lll}0 & 0 & 0.5\end{array}\right), \mathrm{T}$

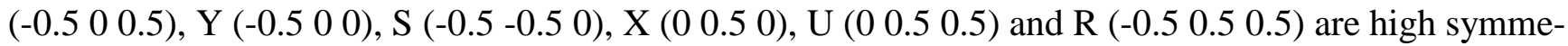
try points in the Brillouin zone. The right panels present the DOS of Pnnm structure projected on the Fe$d$ and N-p orbitals.

\section{Conclusion}

In summary, the stable structures and possible stoichiometries in the Fe- $\mathrm{N}$ system at high pressure are explored by first-principles crystal structure prediction based on PSO algorithm. The Pnnm structure of $\mathrm{FeN}_{2}$, a well-known marcasite phase in the case of transition-metal pernitrides $\mathrm{AN}_{2}$, is predicted to be more stable than the $R-3 m$ structure at pressures above $22 \mathrm{GPa}$. Moreover, its dynamical and mechanical stability under pressure are also verified. The COHP and PDOS analyses demonstrate the pernitride unit is formulated as $(\mathrm{N}=\mathrm{N})^{2-}$ and the mixed covalent $\mathrm{N}-\mathrm{N}$ and $\mathrm{Fe}-\mathrm{N}$ bonding nature are present in the structure. The mechanical properties including bulk moduli, shear moduli, Young's moduli, Poisson's ratio and Vickers hardness have been further computed, revealing that Pnnm structure exhibits excellent mechanical properties, which are correlated with the dinitrogen units.

*Email of corresponding author: cyz@ calypso.org.cn

\section{Acknowledgment}

This work is supported by the Introduce Talents Start Scientific Research Funds of Southwest Jiaotong 
University (A0920502051509-35); the China National Science Foundation (Grant No. 11604270, 11174237, 11404268, 21363013); the Sichuan Province, Applied Science and Technology Project (Grant No. 2013JY0035); the Fundamental Research Funds for the Central Universities (2682014ZT30 and 2682015QM04); the Open Research Fund of Computational Physics Key Laboratory of Sichuan Province, Yibin University (No. 2016H01038); the Open Research Fund of Province of state key laboratory cultivation base construction, Inner Mongolia University of Science \& Technology (No. 2015H01424).

\section{References}

[1] X. Xu, S. A. Shaheen, $a(A), 8$ (1893) 8.

[2] K. H. Jack, Proc. R. Soc. London Ser. A 208 (1951) 200.

[3] K. H. Jack, Proc. R Soc. London Ser. A 195 (1948) 34.

[4] H. Jacobs, D.Rechenbach, and U. Zachwieja, J. Alloys Compd. 227 (1995) 10

[5] K. Suzuki, H. Morita, T. Kaneko, H. Yoshida, and H. Fujimori. J. Alloys. Compd. 201 (1993) 11

[6] M. Wessel and R. Dronskowski, Chem. Eur. J. 17 (2011) 2598

[7] S. D. Gupta, S. K. Gupta, and P. K. Jha, Eur. Phys. J. B 86 (2013) 8

[8] A. F. Young, C. Sanloup, E. Gregoryanz, S. Scandolo, R. J. Hemley, and H. K. Mao, Phys. Rev. Lett. 96 (2006) 155501.

[9] J. C. Crowhurst, A. F. Goncharov, B. Sadigh, J. M. Zaug, D. Aberg, Y. Meng, and V. B. Prakapenka, J. Mater. Res. 23 (2008) 1

[10] W. Zhijian, H. Xianfeng, L. Xiaojuan, M. Jian Meng, Phys. Rev. B 75 (2007) 054115

[11] Z. W. Chen, X. J. Guo, Z. Y. Liu, M. Z. Ma, Q. Jing, G. Li, X. Y. Zhang, L. X. Li, Q. Wang, Y. J. Tian, and R. P. Liu, Phys. Rev. B 75 ( 2007) 054103

[12] K.Niwa, K. Suzuki, S. Muto, K. Tatsumi, K. Soda, T. Kikegawa, M. Hasegawa, Hasegawa M. Chem. Eur. J. $20(2014) 13885$

[13] (a) Y Wang, J. Lv, L. Zhu, Y. Ma, Phys. Rev. B 82 (2010) 094116. (b) Y. Wang, J. Lv, L. Zhu, Y. Ma, Comput. Phys. Commun. 183 (2012) 2063-2070. (c) CALYPSO code is free for academic use, please register at http://www.calypso.cn.

[14] Y. Chen, F. Peng, Y. Yan, Z. Wang, C. Sun, and Y. Ma, J. Phys. Chem. C.117 (2013) 13879

[15] Y. Chen, X. Xi, W. Yim, F. Peng, Y. Wang, H. Wang, Y. Ma, G. Liu, C. Sun, C. Ma, Z. Chen, and H. Berger, J. Phys. Chem. C 117 (2013) 25677

[16] L. Zhu, H. Wang, Y. Wang, J. Lv, Y. Ma, Q. Cui, Y. Ma, and G. Zou, Phys. Rev. Lett. 106 (2011) 145501.

[17] D. Nishio-Hamane, M. Zhang, T. Yagi, and Y. Ma, Am. Mineral. 97 (2012) 568

[18] C. Guillaume, E. Gregoryanz, O. Degtyareva, M. McMahon, M. Hanfland, S. Evans, M. Guthrie, S. Sinogeikin, H. Mao, Nat. Phys. 7 (2011) 211 
[19] H. Wang, J. S. Tse, K. Tanaka, T. Iitaka, and Y. Ma, Proc. Natl. Acad. Sci. USA 109 (2012) 6463

[20] L. Zhu, Z. Wang, Y. Wang, and Y. Ma, Proc. Natl. Acad. Sci. USA 109 (2012) 751

[21] Y. Wang, H. Liu, J. Lv, L. Zhu, H. Wang, and Y. Ma, Nature Commun. 2 (2011) 563.

[22] P. E. Blöchl, Phys. Rev. B 50 (1994) 17953

[23] G. Kresse, and D. Joubert, Phys. Rev. B 59 (1999) 1758

[24] G. Kresse, and J. Furthmüller, PhysRev. B 54 (1996) 11169.

[25] H. J. Monkhorst, and J. D. Pack, Phys. Rev. B 13 (1976) 5188.

[26] K. Parlinski, Z.Q. Li, and Y. Kawazoe, Phys. Rev. Lett. 78 (1997) 4063

[27] W. Zhijian, Z. Er-jun, X. Hongping, H. Xianfeng, L. Xiaojuan and M. Jian, Phys. Rev. B 76 (2007) 054115.

[28] R. Hill, London 65 (1952) 349

[29] X. Q. Chen, H. Y. Niu, D. Z. Li, and Y. Y. Li, 19 (2011) 1275

[30] Y. J. Tian, B. Xu, and Z. S. Zhao, 33 (2012) 93

[31] R. Dronskowski, and P. E. Blöchl, J. Phys. Chem. 97 (1993) 8617

[32] V. L. Deringer, A. L. Tchougréeff, and R. Dronskowski, J. Phys. Chem. A 115 (2011) 5461

[33] O. Brovarets, and D. M. Hovorun, J. Comput. Chem. 34 (2013) 2577

[34] A. F. Young, J. A. Montoya, C. Sanloup, M. Lazzeri, E. Gregoryanz, and S. Scandolo, Phys. Rev. B 73 (2006) 153102

[35] Z. W. Chen, X. J. Guo, Z. Y. Liu, M. Z. Ma, Q. Jing, G. Li, X. Y. Zhang, L. X. Li, Q. Wang, Y. J. Tian, and R. P. Liu, Phys. Rev. B75 (2007) 054103

[36] O. Gomis, R. Vilaplana, et al., J. Appl. Phys. 113 (2013) 073510

[37] F. Mouhat and C. Franc, ois-Xavier, Phys. Rev. B. 90 (2014) 224104

[38] D. Errandonea, Ch. Ferrer-Roca, D. Martínez-Garcia, A. Segura, et al., Phys. Rev. B 82 (2010) 174105

[39] J. C. Crowhurst, A. F. Goncharov, B. Sadigh, C. L. Evans, P. G. Morrall, J. L. Ferreira, and A. J. Nelson, Science 2006, 311, 1275

[40] P. K. Gantzel, N. L. Baldwin, Acta Crystallogr. 17 (1964) 772.

[41] S. B. Schneider, M. Seibald, V. L. Deringer, et al. J. Am. Chem. Soc. 135 (2013) 16668

[42] A, Arnaldsen, W, Tang, G, Henkelman. Bader charge analysis [J]. 2012.

[43] M.Wessel, R. Dronskowski, J. Am. Chem. Soc. 132 (2010) 2421

[44] Z. T. Y. Liu, D. Gall,and S. V. Khare, Phys. Rev. B 90 (2006) 134102

[45] W. Sun, Y. Li, L. Zhu, Y. Ma, I. D Marco, et al., Phys. Chem. Chem. Phys, 17 (2015) 9730 